\title{
Propensity-score adjusted comparison of pathologic nodal upstaging by robotic, video-assisted thoracoscopic, and open lobectomy for non-small cell lung cancer
}

\author{
Peter J. Kneuertz, MD, ${ }^{a}$ Danjouma H. Cheufou, MD, ${ }^{b}$ Desmond M. D'Souza, MD, ${ }^{a}$ \\ Khaled Mardanzai, MD, ${ }^{\mathrm{b}}$ Mahmoud Abdel-Rasoul, MS, MPH, ${ }^{\mathrm{c}}$ Dirk Theegarten, MD, ${ }^{\mathrm{d}}$ \\ Susan D. Moffatt-Bruce, MD, PhD, MBA, ${ }^{a}$ Clemens Aigner, MD, MBA, ${ }^{b}$ and Robert E. Merritt, MD $^{\mathrm{a}}$
}

\section{ABSTRACT}

Objective: To assess the effectiveness of intraoperative lymph node (LN) staging by comparing upstaging between robotic-assisted surgery, video-assisted thoracoscopic surgery (VATS), and open thoracotomy approach for lobectomy for nonsmall cell lung cancer.

Methods: We retrospectively analyzed 1053 patients with clinical stage N0/N1 non-small cell lung cancer who underwent lobectomy at 2 centers between 2011 and 2018. Propensity score adjustment by inverse probability of treatment weighting was used to balance baseline characteristics. The primary end point was LN upstaging.

Results: A total of 911 patients (254 robotic, 296 VATS, and 261 open) were included in the inverse probability of treatment weighting adjusted analysis. The overall rate of LN upstaging was highest with open lobectomy $(21.8 \%)$, followed by robotic $(16.2 \%)$, and VATS $(12.3 \%)(P=.03)$. Mediastinal N2 upstaging was observed in similar frequencies (open $6.9 \%$ vs robotic $6.3 \%$ vs VATS $4.4 \% ; P=.6$ ). No differences were seen for total LN counts, but were observed in the number of stations sampled (mean, open 4.0 vs robotic 3.8 vs VATS 3.6; $P=.001$ ). On multivariate analysis, LN upstaging was lower for VATS compared with open (odds ratio, 0.50; 95\% confidence interval, 0.29-0.85), but not different between robotic and open (odds ratio, $0.72 ; 95 \%$ confidence interval, 0.44-1.18). No significant differences were seen in mediastinal $\mathrm{N} 2$ upstaging between groups.

Conclusions: Pathologic LN upstaging following lobectomy for clinically N0/N1 NSCLC remains high. Compared with a traditional thoracotomy approach, robotic lobectomy was associated with similar and VATS with lower overall nodal upstaging. A thorough evaluation of hilar and mediastinal LNs remains critical to ensure accurate staging by detection of occult LN metastases. (J Thorac Cardiovasc Surg 2019;158:1457-66)

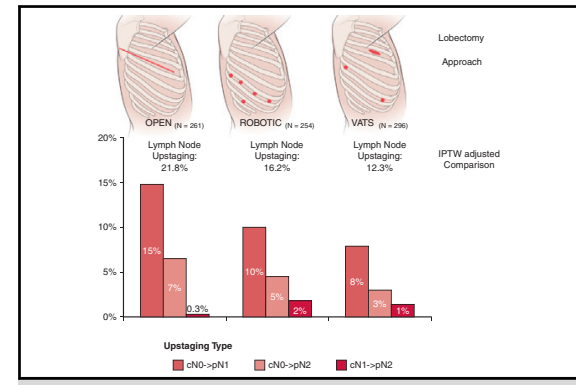

NSCLC lymph node upstaging by lobectomy approach.

\section{Central Message}

Compared with the open lobectomy approach robotic lobectomy is associated with similar and VATS with lower, lymph node assessment and pathologic upstaging.

\section{Perspective}

In a propensity score weighted analysis of a large database from 2 academic centers, we found significant variations in intraoperative lymph node staging of clinically N0/N1 NSCLC patients undergoing lobectomy. Pathologic lymph node upstaging as a marker for completeness of nodal evaluation was significantly lower for VATS compared with the open approach, but similar between robotic and open procedures.

See Commentaries on pages 1467 and 1469.
Intraoperative lymph node assessment is a critical component in the surgical treatment of non-small cell lung cancer (NSCLC). Multiple contemporary studies have emphasized

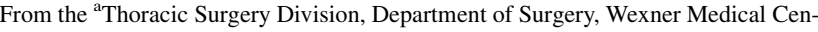
ter, and ${ }^{\mathrm{c}}$ Center for Biostatistics, The Ohio State University, Columbus, Ohio; and ${ }^{b}$ Ruhrlandklinik and ${ }^{\mathrm{d}}$ University Hospital, University of Duisburg-Essen, Essen, Germany.

Drs Kneuertz and Cheufou contributed equally to this work as first author Drs Aigner and Merritt contributed equally to this work as last author

Read at the 99th Annual Meeting of The American Association for Thoracic Surgery, Toronto, Ontario, Canada, May 4-7, 2019.
}

the limitation of clinical staging modalities, such as the use of computed tomography and positron emission tomography (PET) scanning with selective invasive mediastinal

Received for publication Jan 31, 2019; revisions received June 11, 2019; accepted for publication June 17, 2019.

Address for reprints: Peter J. Kneuertz, MD, Thoracic Surgery Division, Department of Surgery, The Ohio State University, Wexner Medical Center, Doan Hall N846, 410 W 10th Ave, Columbus, OH 43210 (E-mail: Peter.Kneuertz@osumc.edu). 0022-5223/ $\$ 36.00$

Copyright (C) 2019 by The American Association for Thoracic Surgery https://doi.org/10.1016/j.jtcvs.2019.06.113 


\section{Abbreviations and Acronyms \\ EBUS = endobronchial ultrasound \\ IPTW = inverse probability of treatment weighting \\ LN $=$ lymph node \\ NSCLC $=$ non-small cell lung cancer \\ PET = positron emission tomography \\ STS = Society of Thoracic Surgeons \\ VATS $=$ video-assisted thoracoscopic surgery}

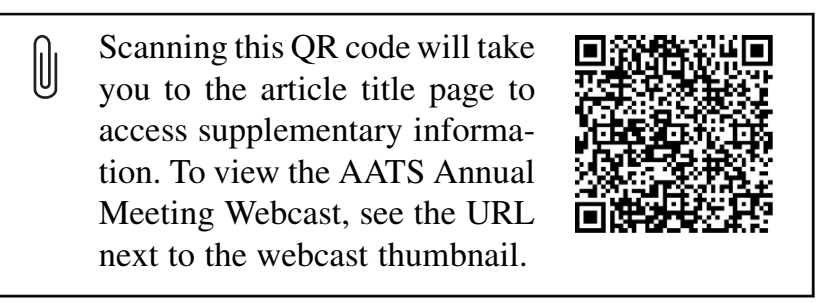

staging because they fall short in identifying occult nodal metastases in $10 \%$ to $24 \%$ of patients with clinically stage I NSCLC. ${ }^{1-4}$ Lymph node (LN) upstaging has therefore emerged as an important surrogate measure of completeness of surgical nodal assessment and a useful end point of oncologic effectiveness when comparing surgical techniques for lung cancer. ${ }^{3,5}$

Over the past decades, the surgical treatment of NSCLC has evolved with increasing use of minimally invasive techniques, first with the use of video-assisted thoracoscopic surgery (VATS) and more recently with robotics. Minimally invasive lobectomy has been associated with improved perioperative and comparable or superior long-term outcomes. ${ }^{6,7}$ However, controversy remains about the $\mathrm{LN}$ assessment. Although previous studies have shown that VATS can yield an adequate lymphadenectomy, ${ }^{8-10}$ other studies have observed that nodal upstaging with VATS was significantly less common. ${ }^{3,5,11}$

A perceived benefit of robotic surgery is the ease of LN dissection. Recent studies have suggested good nodal assessment using the robotic approach; however, comparative data are limited. ${ }^{12-14}$ We hypothesized that based on the current experience, there is no difference in the effectiveness of nodal resection by robotic, VATS, and open lobectomy when adjusting for variables associated with selection of the surgical approach.

The purpose of this study was to compare the quality of surgical LN assessment between the robotic, VATS, and open thoracotomy approach for lobectomy for NSCLC. We aim to estimate true comparative rates of nodal upstaging using a large dataset from 2 academic medical centers with expertise in minimally invasive thoracic surgery, and minimize patient selection bias using a propensity score weighted analysis.

\section{METHODS}

This is a retrospective review of a collaborative database from 2 academic medical centers that was approved by the institutional review board at each institution. We combined all available pulmonary lobectomies performed for clinical stage N0 or N1 NSCLC performed at The Ohio State University Wexner Medical Center (Columbus, Ohio) between 2012 and 2017, and at the Ruhrlandklinik of the University of Duisburg-Essen (Essen, Germany) between years 2015 and 2017, captured in the respective institutional databases. Patients undergoing lobectomy with complete clinical staging information were included. Patients undergoing segmentectomy, pneumonectomy, or nonanatomic resection were excluded. We also excluded patients who received preoperative radiation or systemic therapy. Patients were assigned to comparison groups based on the surgical approach of robotic, VATS, or open thoracotomy. The approach was selected at the discretion of the surgeon. Robotic lobectomy was performed using the port-based 4-instrument technique with an additional assistant port. The DaVinci Si (Intuitive Surgical Inc, Sunnyvale, Calif) robot was used in both institutions and replaced by the Xi model at Wexner Medical Center in 2016. VATS cases were performed by different techniques (4-port closed technique, 3-incision technique with anterior access incision, or uniportal). Anterolateral or posterolateral thoracotomy was performed for open lobectomy cases. The operative volume with robotic and VATS lobectomy varied among locations (Table E1). The majority of robotic and VATS procedures were performed by 6 experienced minimally invasive surgeons: 2 surgeons who primarily performed robotic procedures who had surpassed their learning curve, 2 experienced VATS surgeons who transitioned to robotics within the study period, and 2 primarily VATS surgeons. Another 3 surgeons with less experience in thoracoscopic lobectomy contributed mostly open cases.

\section{Definitions}

Both centers provided data as specified through a menu-driven database, prepared with precise instructions for coding fields and dropdown lists. Definitions of patient demographic characteristics, clinical characteristics, and outcomes were adopted from the Society of Thoracic SurgeonsGeneral Thoracic Surgery Database version 2.3 training manual. ${ }^{15}$ Staging information was provided according to the American Joint Committee on Cancer eighth edition staging manual. Clinical T-stage was assessed based on tumor features on preoperative cross-sectional imaging. Clinical $\mathrm{N}$-stage was determined based on presence or absence of suspicious LNs due to enlarged size $(>1 \mathrm{~cm}$ in short-axis) on computed tomography or hypermetabolic activity on PET scan. The use of invasive preoperative nodal staging by endobronchial ultrasound (EBUS) or mediastinoscopy was recorded, and used based on the guidelines of the American College of Chest Physicians and the European Society of Thoracic Surgeons. Pathologic staging information, margin status, histologic tumor features, and presence of granulomatous LN disease were extracted from the pathology reports.

\section{Assessment of Nodal Upstaging}

The primary end point in this study was the rate of LN upstaging, which was defined as the percentage of patients who had an increase in the $\mathrm{N}$-stage after pathologic evaluation of the surgical specimen. The overall rate of LN upstaging included patients who were found to have occult LN metastases after being clinically staged N0, and patients with clinical N1 disease who were found to have occult mediastinal (N2) LN metastases. The rate of mediastinal $(\mathrm{cNO} / \mathrm{cN} 1 \rightarrow \mathrm{pN} 2)$ upstaging was compared separately. Because the choice of $\mathrm{LN}$ sampling or complete lymphadenectomy was not standardized in this study, we determined the extent of nodal assessment for the 3 surgical approaches based the number of LN stations sampled and LN counts. LN counts were derived from the patients' 
pathology reports and may have included LN fragments labeled as individual LNs. Nodal stations sampled and number of retrieved nodes was recorded and analyzed for the individual nodal stations separately. We grouped adjacent mediastinal LN stations to reduce the possibility of mislabeling, distinguishing 6 surgically resected anatomic nodal clusters: station \#4, stations \#5 and \#6, station \#7, stations \#8 and \#9, station \#10, and station \#11. Nodal upstaging in the individual nodal stations, number of LN stations assessed, and LN counts were secondary endpoints.

\section{Statistical Analysis}

A crude analysis was performed to comparing baseline characteristics, to test for differences between surgery groups. Categorical variables were summarized as frequencies and percentages and compared between surgery groups using $\chi^{2}$ tests or Fisher exact tests where relevant. Continuous baseline characteristics were reported as means \pm standard error and compared using analysis of variance. To account for treatment selection bias and minimize confounding by tumor characteristics, we applied a propensity score methodology with inverse probability of treatment weighing (IPTW) to balance the baseline characteristics between the 3 lobectomy groups, as previously described. ${ }^{6}$ The IPTWs were estimated using multinomial logistic regression analysis, including the following differential patient and disease characteristics $(P \leq .2)$, and disregarding outcomes: age, smoking status, body mass index, coronary artery disease, chronic obstructive lung disease, diabetes mellitus, forced expiratory volume in 1 second, preoperative mediastinal staging by EBUS or mediastinoscopy, the resected lobe, clinical T-stage, clinical N-stage, histology, grade, lymphovascular invasion, visceral pleural invasion, granulomatous LN disease, reoperation, and the center performing the operation. Data for these variables were available for $89.6 \%$ of patients, and were assumed to be missing at random. Stabilized inverse probability of treatment weights were calculated for each patient by dividing the predicted probability of being in the treatment group that a patient is in as estimated from a crude multinomial logistic regression model by their respective predicted probability from the multivariable adjusted multinomial logistic regression model. The stabilized weights for each patient were used as the final IPTWs. The final analysis data set was further trimmed to only include patients with common support of the estimated predicted probability among the 3 surgical procedure groups. Patients with predicted probabilities lower than the minimum or higher than the maximum predicted probability for any other surgical procedure group were excluded. Patient characteristics were then reassessed for balance between surgery groups after adjusting for the IPTWs. Categorical variables and rates of nodal upstaging were compared between surgery groups after adjusting for IPTWs using Rao-Scott $\chi^{2}$ tests. IPTWadjusted linear regression models were used to test for differences in continuous variables. IPTW adjusted multivariable adjusted binary logistic regression models were used to assess the relationship between surgical procedure and each of the respective overall and mediastinal LN upstaging outcomes. Variables that were not properly balanced after IPTW adjustment were further assessed for association with the outcome, potential confounding, and effect modification resulting in the final doubly robust adjusted multivariable binary logistic regression models presented. Patients converted from minimally invasive to an open procedure were compared based on the initial approach according to an intention-to-treat analysis. Hypothesis testing for main outcomes was conducted at a $5 \%$ type I error rate. The statistical analysis was designed and conducted by an experienced and impartial biostatistician (MA) using SAS version 9.4 (SAS Institute Inc, Cary, NC) and Stata version 13 (StataCorp, College Station, Tex).

\section{RESULTS}

\section{Patient and Clinicopathologic Characteristics}

A total of 1053 patients were identified who underwent lobectomy for clinical stage N0/N1 NSCLC, including
391 in the robotic, 338 in the VATS, and 324 in the open thoracotomy group. A Consolidated Standards of Reporting Trials diagram detailing the patient selection is provided in Figure E1. A comparison of patients' demographic characteristics and clinical features is provided in Table 1 . Before propensity score weighting, the groups differed in multiple baseline characteristics (Table 1). Differences were also seen in the unadjusted comparison of pathologic features of the resected tumors among the 3 groups, which are summarized in Table 2. Propensity scores were then utilized through the method of IPTW adjustment to properly assess differences in outcomes between properly balanced surgical approach groups. During this process, we excluded additional patients who were lacking common support, or were missing the comparative data (Figure E1). Finally, the IPTW-weighted cohort included 911 patients (254 robotic, 296 VATS, and 261 open). Following application of IPTWs, the 3 groups were well balanced, with similar distribution of patients' baseline characteristics (Table 1). The use of preoperative mediastinal staging by EBUS or mediastinoscopy remained higher in the VATS and open groups compared with the robotic group ( $32 \%$ vs $29 \%$ vs $16 \%$, respectively; $P=.001$ ), owing to variations in staging and patterns between the 2 institutions (Table E1). Pathologic tumor features were well balanced among the robotic, VATS, and open thoracotomy groups in the weighted cohorts, including similar distribution of histology, tumor size, pathologic T-stage, grade, and presence of lymphovascular invasion (Table 2).

\section{Comparison of Nodal Assessment}

The nodal assessment by robotic, VATS, and open lobectomy was analyzed in the IPTW-weighted groups (Table 3). There was no difference in the sum of $\mathrm{N} 1$ and/or $\mathrm{N} 2$ nodes harvested between the groups (Table 3 ). When comparing the number of individual nodal stations $(\mathrm{N} 1+\mathrm{N} 2)$ that were sampled, the groups varied. The lowest number of nodal stations were harvested in the VATS group, followed by robotic surgery group, and highest in the open thoracotomy group (mean, robotic 3.8 vs VATS 3.6 vs open 4.0; $P=.001$ ). A complete breakdown of the number of nodal stations sampled by approach is summarized in Table E2. Three or more N2 stations were recorded in $60 \%$ of the robotic, $53 \%$ of the open lobectomy, and $37 \%$ of VATS lobectomy groups $(P<.001)$. Differences in the sampling rates of individual nodal stations are summarized in Table 3 .

\section{Comparison of Upstaging}

The IPTW-adjusted pathologic nodal upstaging was highest in the open thoracotomy group with an overall rate of $21.8 \%$, followed by $16.2 \%$ in the robotic and $12.3 \%$ in the VATS groups $(P=.03)$. Upstaging was most frequently seen in clinically node-negative patients who were found to have occult N1 disease (N1 upstaging, 
TABLE 1. Patient and clinical baseline characteristics in unadjusted and inverse probability treatment weight (IPTW)-adjusted cohorts

\begin{tabular}{|c|c|c|c|c|c|c|c|c|}
\hline \multirow[b]{2}{*}{ Characteristic } & \multicolumn{4}{|c|}{ Without IPTW } & \multicolumn{4}{|c|}{ With IPTW } \\
\hline & $\begin{array}{c}\text { Robotic } \\
(\mathrm{n}=391)\end{array}$ & $\begin{array}{c}\text { VATS } \\
(\mathbf{n}=\mathbf{3 3 8})\end{array}$ & $\begin{array}{c}\text { Open } \\
(n=324)\end{array}$ & $P$ value & $\begin{array}{c}\text { Robotic } \\
(n=254)\end{array}$ & $\begin{array}{c}\text { VATS } \\
(\mathbf{n}=\mathbf{2 9 6})\end{array}$ & $\begin{array}{c}\text { Open } \\
(n=261)\end{array}$ & $P$ value \\
\hline Age (y) & $65.9 \pm 0.5$ & $64.4 \pm 0.5$ & $65.2 \pm 0.6$ & .09 & $65.2 \pm 0.6$ & $64.7 \pm 0.7$ & $64.3 \pm 0.7$ & .53 \\
\hline \multicolumn{9}{|l|}{ Sex } \\
\hline Female & $221(56.5)$ & $179(53)$ & $141(43.5)$ & .002 & $54 \%$ & $53 \%$ & $51 \%$ & .74 \\
\hline Male & $170(43.5)$ & $159(47)$ & $183(56.5)$ & & $46 \%$ & $47 \%$ & $49 \%$ & \\
\hline \multicolumn{9}{|l|}{ Smoking status } \\
\hline Never & $62(16.7)$ & 49 (15.6) & $33(11.2)$ & .20 & $14 \%$ & $16 \%$ & $14 \%$ & .96 \\
\hline Former & $180(48.5)$ & $146(46.3)$ & $158(53.6)$ & & $50 \%$ & $47 \%$ & $50 \%$ & \\
\hline Active & $129(34.8)$ & $120(38.1)$ & $104(35.3)$ & & $36 \%$ & $37 \%$ & $36 \%$ & \\
\hline Total & 371 & 315 & 295 & & & & & \\
\hline BMI & $27.9 \pm 0.3$ & $26.9 \pm 0.3$ & $27.3 \pm 0.3$ & .13 & $27.6 \pm 0.4$ & $27.4 \pm 0.4$ & $27.4 \pm 0.5$ & .88 \\
\hline \multicolumn{9}{|l|}{ ECOG performance status } \\
\hline 0 & $137(37.4)$ & $142(50)$ & $131(48.2)$ & .018 & $38 \%$ & $48 \%$ & $49 \%$ & .35 \\
\hline 1 & $190(51.9)$ & $125(44)$ & $122(44.9)$ & & $53 \%$ & $44 \%$ & $44 \%$ & \\
\hline 2 & $37(10.1)$ & $15(5.3)$ & $17(6.3)$ & & $9 \%$ & $7 \%$ & $6 \%$ & \\
\hline 3 & $2(0.5)$ & $2(0.7)$ & $2(0.7)$ & & $1 \%$ & $1 \%$ & $1 \%$ & \\
\hline Total & 336 & 284 & 272 & & & & & \\
\hline Rheumatoid arthritis & $5(1.3)$ & $4(1.2)$ & $1(0.3)$ & .39 & $1 \%$ & $1 \%$ & $0 \%$ & .52 \\
\hline Diabetes mellitus & $50(12.8)$ & $28(8.3)$ & $24(7.4)$ & .030 & $12 \%$ & $11 \%$ & $9 \%$ & .64 \\
\hline Coronary artery disease & $142(36.2)$ & $76(22.5)$ & $110(34)$ & $<.001$ & $39 \%$ & $33 \%$ & $36 \%$ & .24 \\
\hline COPD & $161(41.2)$ & 107 (31.7) & $112(34.6)$ & .022 & $40 \%$ & $40 \%$ & $36 \%$ & .78 \\
\hline FEV1 (\% predicted) & $82.8 \pm 1.0$ & $82.5 \pm 1.1$ & $77.5 \pm 1.1$ & .006 & $81.8 \pm 1.2$ & $81.2 \pm 1.2$ & $80.3 \pm 1.7$ & .74 \\
\hline DLCO $(\%$ predicted $)$ & $78.0 \pm 1.1$ & $75.7 \pm 1.2$ & $70.3 \pm 1.2$ & $<.001$ & $77.2 \pm 1.4$ & $77.8 \pm 1.4$ & $73.0 \pm 1.6$ & .07 \\
\hline Reoperation & $7(1.8)$ & $8(2.4)$ & $20(6.2)$ & .002 & $3 \%$ & $3 \%$ & $3 \%$ & .98 \\
\hline \multicolumn{9}{|l|}{ Anatomic location } \\
\hline Right upper lobe & $124(31.7)$ & $122(36.1)$ & $127(39.2)$ & .18 & $35 \%$ & $36 \%$ & $37 \%$ & .96 \\
\hline Right middle lobe & $28(7.2)$ & $23(6.8)$ & $23(7.1)$ & & $4 \%$ & $9 \%$ & $6 \%$ & \\
\hline Right lower lobe & $89(22.8)$ & $57(16.9)$ & $66(20.4)$ & & $21 \%$ & $19 \%$ & $19 \%$ & \\
\hline Left upper lobe & $94(24)$ & $83(24.6)$ & $77(23.8)$ & & $24 \%$ & $24 \%$ & $28 \%$ & \\
\hline Left lower lobe & $56(14.3)$ & $53(15.7)$ & $31(9.6)$ & & $13 \%$ & $12 \%$ & $11 \%$ & \\
\hline PET scan & $359(91.8)$ & $267(79.5)$ & $250(77.2)$ & $<.001$ & $92 \%$ & $86 \%$ & $86 \%$ & .051 \\
\hline \multicolumn{9}{|c|}{ Invasive mediastinal staging } \\
\hline EBUS & $68(17.4)$ & 117 (34.6) & $140(43.2)$ & $<.001$ & $16 \%$ & $32 \%$ & $29 \%$ & .001 \\
\hline Mediastinoscopy & $22(5.6)$ & $25(7.4)$ & $42(13)$ & .001 & $7 \%$ & $9 \%$ & $11 \%$ & .50 \\
\hline \multicolumn{9}{|l|}{ Clinical T-stage } \\
\hline 1 & $281(71.9)$ & $209(61.8)$ & $139(43)$ & $<.001$ & $69 \%$ & $63 \%$ & $61 \%$ & .20 \\
\hline 2 & $95(24.3)$ & $113(33.4)$ & $134(41.5)$ & & $27 \%$ & $31 \%$ & $32 \%$ & \\
\hline 3 & $15(3.8)$ & $11(3.3)$ & $41(12.7)$ & & $4 \%$ & $4 \%$ & $6 \%$ & \\
\hline 4 & 0 & $5(1.5)$ & $9(2.8)$ & & $0 \%$ & $2 \%$ & $1 \%$ & \\
\hline \multicolumn{9}{|l|}{ Clinical N-stage } \\
\hline 0 & 374 (95.7) & 315 (93.2) & $278(85.8)$ & $<.001$ & $93 \%$ & $93 \%$ & $92 \%$ & .81 \\
\hline 1 & $17(4.3)$ & $23(6.8)$ & $46(14.2)$ & & $7 \%$ & $7 \%$ & $8 \%$ & \\
\hline \multicolumn{9}{|l|}{ Institution } \\
\hline Center 1 & $37(9.5)$ & $162(47.9)$ & $145(44.8)$ & $<.001$ & $7 \%$ & $26 \%$ & $28 \%$ & $<.001$ \\
\hline Center 2 & $354(90.5)$ & $176(52.1)$ & $179(55.2)$ & & $93 \%$ & $74 \%$ & $72 \%$ & \\
\hline
\end{tabular}

Values are presented as mean \pm standard error, $\mathrm{n}(\%)$, or \%. Significant $P$ values are in bold. IPTW, Inverse probability treatment weight; VATS, video-assisted thoracoscopic surgery; $B M I$, body mass index; $E C O G$, Eastern Cooperative Oncology Group; $C O P D$, chronic obstructive pulmonary disease; $F E V 1$, forced expiratory volume in 1 second; $D L C O$, diffusing capacity of carbon monoxide; PET, positron emission tomography; EBUS, endobronchial ultrasound. 
TABLE 2. Pathologic details of patients with non-small cell lung cancer in unadjusted cohorts and cohorts adjusted for inverse probability treatment weight (IPTW)

\begin{tabular}{|c|c|c|c|c|c|c|c|c|}
\hline \multirow[b]{2}{*}{ Detail } & \multicolumn{4}{|c|}{ Without IPTW } & \multicolumn{4}{|c|}{ With IPTW } \\
\hline & $\begin{array}{c}\text { Robotic } \\
(n=391)\end{array}$ & $\begin{array}{c}\text { VATS } \\
(\mathbf{n}=338)\end{array}$ & $\begin{array}{c}\text { Open } \\
(n=324)\end{array}$ & $P$ value & $\begin{array}{c}\text { Robotic } \\
(n=254)\end{array}$ & $\begin{array}{c}\text { VATS } \\
(n=296)\end{array}$ & $\begin{array}{c}\text { Open } \\
(n=261)\end{array}$ & $P$ value \\
\hline Histology & & & & & & & & .66 \\
\hline Adenocarcinoma & $247(63.2)$ & $213(63)$ & $141(43.5)$ & $<.001$ & $62 \%$ & $58 \%$ & $56 \%$ & \\
\hline Squamous cell & $80(20.5)$ & $72(21.3)$ & $111(34.3)$ & & $20 \%$ & $24 \%$ & $25 \%$ & \\
\hline Mixed/large cell/other & $64(16.4)$ & $53(15.7)$ & $72(22.2)$ & & $18 \%$ & $18 \%$ & $19 \%$ & \\
\hline Tumor size $(\mathrm{cm})$ & $3.0 \pm 0.1$ & $2.9 \pm 0.1$ & $3.8 \pm 0.1$ & $<.001$ & $3.1 \pm 0.2$ & $3.1 \pm 0.1$ & $3.2 \pm 0.1$ & .84 \\
\hline Grade & & & & & & & & .51 \\
\hline Well differentiated & $79(20.3)$ & $31(9.2)$ & $32(10)$ & $<.001$ & $16 \%$ & $15 \%$ & $11 \%$ & \\
\hline Moderately differentiated & $130(33.3)$ & $128(38)$ & $103(32.3)$ & & $34 \%$ & $35 \%$ & $33 \%$ & \\
\hline Poorly/undifferentiated & $181(46.4)$ & $178(52.8)$ & $184(57.7)$ & & $49 \%$ & $50 \%$ & $56 \%$ & \\
\hline Total & 390 & 337 & & & & & & \\
\hline Lymphovascular invasion & $119(30.4)$ & $70(20.7)$ & $84(36.2)$ & .012 & $31 \%$ & $31 \%$ & $28 \%$ & .70 \\
\hline Visceral pleural invasion & $112(28.6)$ & $98(29)$ & $74(23)$ & .15 & $29 \%$ & $27 \%$ & $27 \%$ & .92 \\
\hline Granulomatous lymph node disease & $89(22.8)$ & $46(13.6)$ & $50(15.5)$ & .003 & $25 \%$ & $25 \%$ & $18 \%$ & .15 \\
\hline \multicolumn{9}{|l|}{ Pathologic T-stage } \\
\hline 1 & $180(46)$ & $160(47.3)$ & $118(36.4)$ & $<.001$ & $46 \%$ & $44 \%$ & $45 \%$ & .45 \\
\hline 2 & $149(38.1)$ & $144(42)$ & $122(38)$ & & $36 \%$ & $43 \%$ & $35 \%$ & \\
\hline 3 & 49 (12.5) & $28(8.3)$ & $59(18.4)$ & & $14 \%$ & $11 \%$ & $16 \%$ & \\
\hline 4 & $13(3.3)$ & $6(1.8)$ & $22(6.9)$ & & $5 \%$ & $3 \%$ & $4 \%$ & \\
\hline
\end{tabular}

Values are presented as mean \pm standard error, $\mathrm{n}(\%)$, or $\%$. Significant $P$ values are in bold. IPTW, Inverse probability treatment weight; VATS, video-assisted thoracoscopic surgery.

open $15 \%$ vs robotic $10 \%$ vs VATS $8 \%$ ). Mediastinal (N2) upstaging in clinically $\mathrm{N} 0$ patients was observed following $7 \%$ of open, $5 \%$ of robotic, and $3 \%$ of VATS lobectomies, and less often in patients clinically staged N1 (Figure 1). The rate of nodal upstaging in clinically node-negative patients was lower in patients with $\mathrm{T} 1$ tumors $(15.4 \%)$ compared with patients with T2 tumors $(21 \%)$ (Figure E2). The distribution of upstaging in the individual nodal stations is presented in Figure 2. Although the individual rate of upstaging varied between groups, subcarinal upstaging was the only station that was statistically different (Figure 2). On multivariable analysis, adjusting for patients' clinical and pathologic differences using IPTWs, as well as the use of invasive preoperative mediastinal staging and the center in which the procedure was performed, we found no significant difference of robotic compared with open lobectomy (odds ratio [OR], 0.72; 95\% confidence interval [CI], 0.44-1.18) or VATS lobectomy and robotic lobectomy (OR, 0.70; 95\% CI, 0.401.20). VATS remained associated with less nodal upstaging compared with open thoracotomy with an OR of $0.50(95 \%$ CI, 0.29-0.85). There were no significant interactions between the surgical approach and either invasive mediastinal staging or institution on upstaging, and therefore the final model only included main effects. There was no difference in mediastinal (N2) upstaging on multivariable analysis among the 3 groups (Table 4 ).

\section{DISCUSSION}

In this study, we analyzed a large collaborative database from 2 institutions to compare the quality of nodal assessment between robotic, VATS, and open lobectomy for clinically N0/N1 NSCLC. The propensity score-weighted analysis showed that LN upstaging was significantly lower for VATS lobectomy compared with open thoracotomy. Pathologic LN upstaging following robotic lobectomy was proportionally closer the open approach, but not statistically different from either open or VATS approach. Mediastinal (N2) lymph node upstaging, was not significant different among the 3 groups. However, patients undergoing VATS had significantly less upstaging in the subcarinal area.

The adequacy of LN dissection with minimally invasive thoracoscopic resections has been controversial for as long as they have existed. Most recently, the use of robotic lung surgery is increasing rapidly, and several studies have reported good experiences with $\mathrm{LN}$ dissection, and nodal upstaging ranging from $10 \%$ to $16.4 \%$. $^{12-14,16,17}$ The relative ease of $\mathrm{LN}$ dissection is credited to the high-definition robotic camera and the wristed robotic instrumentation with increased freedom of motion and maneuverability in the chest. ${ }^{18}$ In a multi-institutional effort, Wilson and colleagues ${ }^{14}$ reported a rate of nodal upstaging of $10.9 \%$ for clinical stage I NSCLC that they compare favorably to historic VATS data. ${ }^{14} \mathrm{~A}$ smaller study 
TABLE 3. Inverse probability treatment weight-adjusted comparison of lymph node (LN) assessment by LN count and nodal stations

\begin{tabular}{|c|c|c|c|c|}
\hline Variable & Robotic $(n=254)$ & $\begin{array}{c}\text { Video-assisted } \\
\text { thoracoscopic surgery } \\
(\mathrm{n}=\mathbf{2 9 6})\end{array}$ & $\begin{array}{c}\text { Open } \\
(n=261)\end{array}$ & $P$ value \\
\hline \multicolumn{5}{|l|}{ Paratracheal LNs (\#4) } \\
\hline LN count & $1.23(0.76-1.72)$ & $1.55(1.24-7.85)$ & $1.96(1.54-2.38)$ & .08 \\
\hline Frequency assessed & 52.8 & 47.1 & 59.0 & .04 \\
\hline \multicolumn{5}{|l|}{ Aortopulmonary window LNs (\#5-\#6) } \\
\hline LN count & $0.69(0.44-0.95)$ & $0.62(0.46-0.78)$ & $0.53(0.39-0.66)$ & .43 \\
\hline Frequency assessed & 31.2 & 29.9 & 33.6 & .72 \\
\hline \multicolumn{5}{|l|}{ Subcarinal LNs (\#7) } \\
\hline LN count & $1.93(1.70-2.18)$ & $1.94(1.70-2.18)$ & $1.77(1.54-2.01)$ & .55 \\
\hline Frequency assessed & 85.1 & 78.1 & 85.6 & .06 \\
\hline \multicolumn{5}{|c|}{ Pulmonary ligament/paraesophageal LNs (\#8-\#9) } \\
\hline LN count & $1.42(1.24-1.60)$ & $1.12(0.95-1.30)$ & $1.04(0.89-1.20)$ & .06 \\
\hline Frequency assessed & 72.3 & 59.6 & 60.5 & .009 \\
\hline \multicolumn{5}{|l|}{ Hilar LNs (\#10) } \\
\hline LN count & $1.36(1.21-1.52)$ & $0.87(0.73-1.02)$ & $1.56(1.35-1.77)$ & $<.001$ \\
\hline Frequency assessed & 68.3 & 52.8 & 64.4 & $<.001$ \\
\hline \multicolumn{5}{|l|}{ Interlobar (\#11) } \\
\hline LN count & $2.21(1.87-2.57)$ & $3.39(2.88-3.91)$ & $2.43(2.07-2.79)$ & .001 \\
\hline Frequency assessed & 62.4 & 83.5 & 79.6 & $<.001$ \\
\hline \multicolumn{5}{|l|}{ Total LN counts } \\
\hline N1 count & $6.5 \pm 0.3$ & $6.4 \pm 0.3$ & $6.5 \pm 0.3$ & .97 \\
\hline N2 count & $5.3 \pm 0.3$ & $5.3 \pm 0.3$ & $5.4 \pm 0.3$ & .96 \\
\hline Total count & $11.8 \pm 0.4$ & $11.8 \pm 0.4$ & $11.9 \pm 0.5$ & .95 \\
\hline Total No. of N1+N2 LN stations assessed & $3.8 \pm 0.07$ & $3.6 \pm 0.08$ & $4.0 \pm 0.08$ & .001 \\
\hline
\end{tabular}

Values are presented as mean (95\% confidence interval), $\%$, or mean \pm standard error. Significant $P$ values are in bold. $L N$, Lymph node

by Lee and colleagues ${ }^{12}$ found a similar rate of nodal upstaging between robotic $(13 \%)$ and VATS $(15 \%)$ lobectomy. Our current study is important because it is the first comparative study that showed a similar rate of
LN assessment by pathologic LN upstaging, and completeness of LN evaluation by robotic lobectomy compared with the traditional standard of open lobectomy. However, a significant difference in LN upstaging compared with the

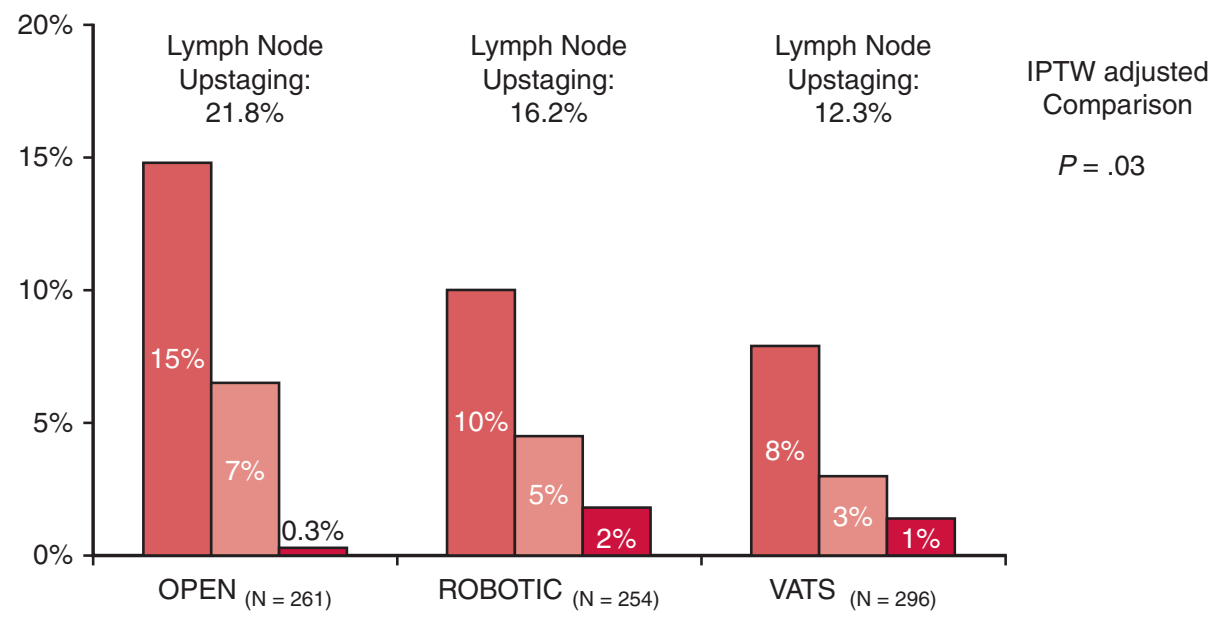

Upstaging Type

$\mathrm{cNO}->\mathrm{pN} 1$

$\mathrm{cNO}->\mathrm{pN} 2$

$\mathrm{cN} 1->\mathrm{pN} 2$

FIGURE 1. Comparison of inverse probability of treatment weight (IPTW) adjusted rates of overall lymph node upstaging for clinical stage N0/N1 nonsmall cell lung cancer by lobectomy approach: open $21.8 \%$ versus robotic $16.2 \%$ versus video-assisted thoracoscopic $($ VATS $) 12.3 \%(P=.03)$. 


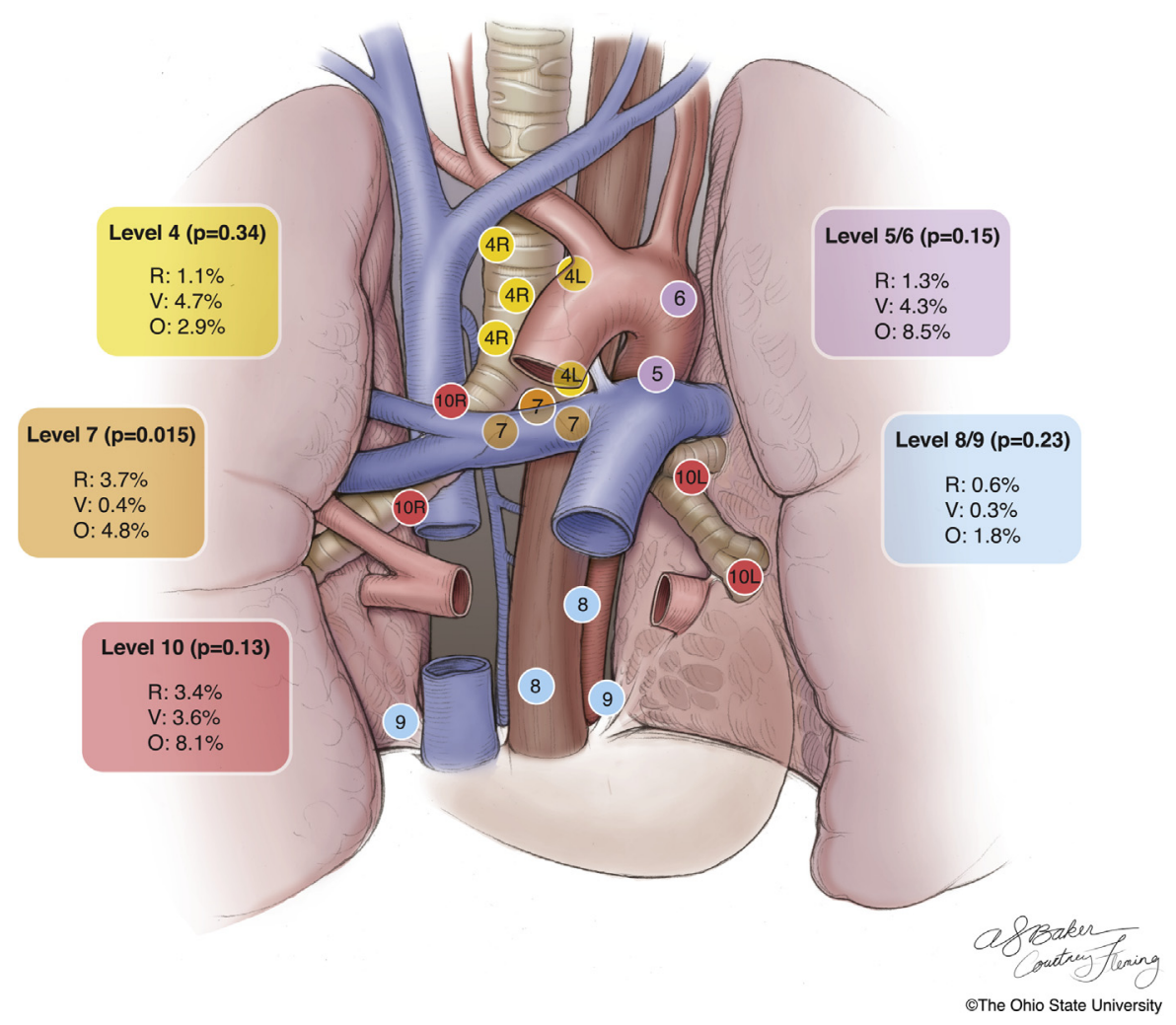

FIGURE 2. Comparison of pathologic lymph node upstaging-specific to mediastinal lymph node stations following lobectomy for clinical N0/N1 nonsmall cell lung cancer, comparing robotic $(R)$, video-assisted thoracoscopic surgery $(V)$, and open thoracotomy $(O)$. Illustration reproduced with the permission of The Ohio State University.

VATS approach was also not observed. The rate of detection of occult $\mathrm{N} 1$ and $\mathrm{N} 2$ disease of $10 \%$ and $4.5 \%$ of clinically node-negative patients in this study is consistent with previous studies ranging from $6.6 \%$ to $9.4 \%$ and $2 \%$ to $6.8 \%$, making these reliable numbers. ${ }^{12-14,16,17}$

The rate of overall ( $\mathrm{N} 1$ and $\mathrm{N} 2$ ) LN upstaging staging following VATS lobectomy in this study was $12.3 \%$, which is in also in keeping with that of most previous studies ranging from $10 \%$ to $15 \%{ }^{3,5,12,19}$ The comparative results

TABLE 4. Multivariate binary logistic regression analysis of nodal upstaging comparing surgical approaches*

\begin{tabular}{lcc}
\hline \multicolumn{1}{c}{ Approach } & $\begin{array}{c}\text { Odds ratio (Adjusted 95\% } \\
\text { confidence interval) } \dagger\end{array}$ & $\begin{array}{c}\text { Adjusted } \\
\boldsymbol{P} \text { value } \dagger\end{array}$ \\
\hline Overall upstaging & & \\
Robotic vs open & $0.716(0.436-1.175)$ & .19 \\
VATS vs open & $0.498(0.293-0.845)$ & $\mathbf{. 0 1}$ \\
VATS vs robotic & $0.695(0.404-1.197)$ & .18 \\
Mediastinal upstaging & & \\
Robotic vs open & $0.856(0.355-2.057)$ & .73 \\
VATS vs open & $0.623(0.259-1.498)$ & .29 \\
VATS vs robotic & $0.729(0.308-1.725)$ & .47 \\
\hline
\end{tabular}

Significant $P$ values are in bold. VATS, Video-assisted thoracoscopic surgery. *Model adjusted for inverse probability of treatment weight, use of invasive mediastinal staging (endobronchial ultrasound or mediastinoscopy), and center performing the operation. $\nmid$ Bonferroni method was used to adjust for multiple comparisons. to open lobectomy also mirror that of multiple previous studies. Lower rates of upstaging for VATS lobectomy compared with the traditional open thoracotomy approach was shown in the review of 3 national datasets, including the Society of Thoracic Surgeons (STS) General Thoracic Surgery database, the National Cancer Database, and the Danish Lung Cancer Registry. ${ }^{3,5,11}$ Although there is a multitude of contributing factors, including the difference in clinical staging and disease factors, we believe that surgical factors likely play a role. Specifically, the completeness of a lymphadenectomy may be influenced by a surgeon's mindset and the perceived added challenges based on the instrumentation and experience level. In an analysis of the STS national database of cases performed between 2001 and 2010, Boffa and colleagues ${ }^{5}$ elegantly demonstrated that the frequency of upstaging increased with the operative volume of VATS. Although the overall LN upstaging with VATS was significantly lower than with open lobectomy in the STS database $(11.6 \%$ vs $14.3 \%)$, there was no difference between VATS and open in centers performing $>80 \%$ of procedures thoracoscopically. Nodal evaluation with a VATS approach is arguably more dependent on the experience and motivation of the surgeon because most surgeons would agree that a thorough $\mathrm{LN}$ dissection is technically more challenging and time-intensive compared 
with thoracotomy. However, even in experienced hands, VATS has been found to result in lower rates of nodal upstaging, such as in the study by Merritt and colleagues, ${ }^{19}$ who reported upstaging in $10.5 \%$ versus $24.6 \%$ by VATS and thoracotomy approach, respectively. Although the LN counts were similar among groups, our study also examined the frequency in which different nodal stations were sampled. Our data showed that the frequency of sampling at least 3 mediastinal LN stations was significantly lower with VATS compared with robotic and open lobectomy, which could be related to an increased effort of nodal dissection with VATS. One should remember that the individual LN counts, and stations sampled, are not ideal end points and should be interpreted with caution. These can be influenced by the variations in intraoperative labeling of $\mathrm{LN}$ location by the surgeon, and counting LN fragments. We therefore chose LN upstaging as the primary end point, which we believe is a more reliable marker of the quality of $\mathrm{LN}$ assessment. Although there was no overall difference in mediastinal upstaging among groups, we found the lowest rate of upstaging of subcarinal LNs in the VATS group. In a previous study from the early VATS experience, Denlinger and colleagues $^{20}$ showed that significantly fewer LNs were removed from the subcarinal space with VATS compared with thoracotomy. Located in the posterior mediastinum, the subcarinal space is perhaps the most difficult area to dissect by VATS, when dissecting from an anterior access incision. Anecdotally, this was found to be easier with the uniportal approach, which is more similar to the situation of a classical anterolateral thoracotomy.

Particular strengths of this study are the large study size, the contribution of 2 centers, and advanced statistical methods using IPTW weighing of propensity scores to adjust for patient selection. We have made every effort to control for possible confounders and effect modifiers, including patient factors, disease characteristics, and other factors that have been found to influence clinical LN staging, such as granulomatous $\mathrm{LN}$ disease. ${ }^{21} \mathrm{~A}$ few patients had missing data and were excluded during this process. When interpreting the results, it is important to acknowledge several limitations related to the retrospective nature of the study. The extent of lymphadenectomy was not standardized, and our comparative results merely reflect how this group of surgeons applied the individual surgical approach to achieve adequate node assessment. The VATS approach was also not standardized. We showed a significantly lower rate of preoperative invasive mediastinal staging in robotic surgery patients, which is largely based on differences in institutional practices with higher use of robotics in 1 center, and overall more frequent use of invasive mediastinal staging but less frequent PET scans in the other center (Table E1). Although mediastinal staging was controlled for in the multivariable analysis, this may still account for some of the observed differences in nodal upstaging despite a similar number of procured LNs with both minimally invasive approaches. The institutional differences did not modify the relationship between surgical approach and upstaging. We cannot completely eliminate selection bias and unmeasured confounders. Specifically, the presence of a large central tumor or other salient features of advanced tumors could influence the choice of thoracotomy over a minimally invasive approach, and influence the likelihood of nodal upstaging. Although we were unable to determine the centrality of tumors in our database, we accounted for the use of invasive staging procedures, squamous histology and tumor stage, which are closely related to central tumor location. ${ }^{22}$ Other potential confounders include aggressive tumor features, such as the micropapillary type adenocarcinoma, and high uptake on PET scan, which we did not routinely capture. Nevertheless, the oncologic principles of $\mathrm{LN}$ harvest and procedure handling were consistent between contributing centers, and the robust statistical analysis allowed us to factor all differing variables in the comparison. Within the limitation of a retrospective analysis, these data provide the best possible estimates of the effectiveness of LN upstaging by robotic surgery and VATS compared with open lobectomy. We did not assess the effect of quality of nodal dissection and upstaging on long-term disease control and survival due to short follow-up time. We plan to address this in a future study, once follow-up has matured.

\section{CONCLUSIONS}

We found significant variation in the completeness of intraoperative LN staging among 3 surgical approaches for pulmonary lobectomy for NSCLC. The number of LNs sampled was comparable for all approaches. The VATS approach was associated with a lower rate of upstaging compared with open thoracotomy. Although the estimated rate of LN upstaging with robotic lobectomy was lower than with open thoracotomy and higher than with VATS, it was not significantly different from either group. A thorough LN evaluation remains critical regardless of surgical approach.

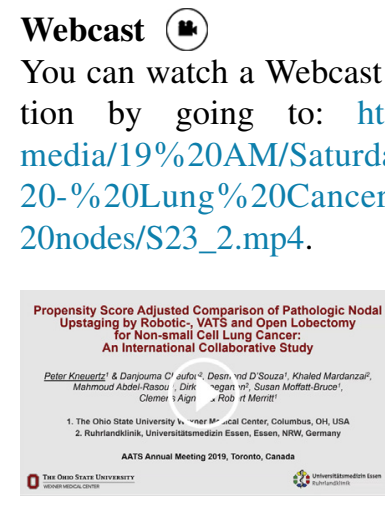




\section{Conflict of Interest Statement}

Dr Merritt has received speakers fees from, and Drs D'Souza and Cheufou are proctors with Intuitive Surgical Inc, Sunnyvale, Calif. All other authors have nothing to disclose with regard to commercial support.

\section{References}

1. Bott MJ, Patel AP, Crabtree TD, Colditz GA, Kreisel D, Krupnick AS, et al. Pathologic upstaging in patients undergoing resection for stage i non-small cell lung cancer: are there modifiable predictors? Ann Thorac Surg. 2015;100:2048-53.

2. Stiles BM, Servais EL, Lee PC, Port JL, Paul S, Altorki NK. Point: clinical stage IA non-small cell lung cancer determined by computed tomography and positron emission tomography is frequently not pathologic IA non-small cell lung cancer: the problem of understaging. J Thorac Cardiovasc Surg. 2009;137:13-9.

3. Licht PB, Jorgensen OD, Ladegaard L, Jakobsen E. A national study of nodal upstaging after thoracoscopic versus open lobectomy for clinical stage I lung cancer. Ann Thorac Surg. 2013;96:943-9.

4. Cerfolio RJ, Bryant AS, Ojha B, Eloubeidi M. Improving the inaccuracies of clinical staging of patients with NSCLC: a prospective trial. Ann Thorac Surg. 2005; 80:1207-13.

5. Boffa DJ, Kosinski AS, Paul S, Mitchell JD, Onaitis M. Lymph node evaluation by open or video-assisted approaches in 11,500 anatomic lung cancer resections. Ann Thorac Surg. 2012;94:347-53.

6. Kneuertz PJ, Singer E, D’Souza DM, Abdel-Rasoul M, Moffatt-Bruce SD, Merritt RE. Hospital cost and clinical effectiveness of robotic-assisted vs. video-assisted thoracoscopic and open lobectomy: a propensity score weighted comparison. J Thorac Cardiovasc Surg. 2019;157:2018-26.e2.

7. Berry MF, Villamizar-Ortiz NR, Tong BC, Burfeind WR Jr, Harpole DH, D'Amico TA, et al. Pulmonary function tests do not predict pulmonary complications after thoracoscopic lobectomy. Ann Thorac Surg. 2010;89:1044-51.

8. D'Amico TA, Niland J, Mamet R, Zornosa C, Dexter EU, Onaitis MW. Efficacy of mediastinal lymph node dissection during lobectomy for lung cancer by thoracoscopy and thoracotomy. Ann Thorac Surg. 2011;92:226-31.

9. Sagawa M, Sato M, Sakurada A, Matsumura Y, Endo C, Handa M, et al. A prospective trial of systematic nodal dissection for lung cancer by video-assisted thoracic surgery: can it be perfect? Ann Thorac Surg. 2002;73:900-4.

10. Watanabe A, Koyanagi T, Ohsawa H, Mawatari T, Nakashima S, Takahashi N, et al. Systematic node dissection by VATS is not inferior to that through an open thoracotomy: a comparative clinicopathologic retrospective study. Surgery. 2005;138:510-7.

11. Medbery RL, Gillespie TW, Liu Y, Nickleach DC, Lipscomb J, Sancheti MS, et al. Nodal upstaging is more common with thoracotomy than with VATS during lobectomy for early-stage lung cancer: an analysis from the National Cancer Data Base. J Thorac Oncol. 2016;11:222-33.

12. Lee BE, Shapiro M, Rutledge JR, Korst RJ. Nodal upstaging in robotic and video assisted thoracic surgery lobectomy for clinical N0 lung cancer. Ann Thorac Surg. 2015;100:229-33.

13. Toosi K, Velez-Cubian FO, Glover J, Ng EP, Moodie CC, Garrett JR, et al. Upstaging and survival after robotic-assisted thoracoscopic lobectomy for nonsmall cell lung cancer. Surgery. 2016;160:1211-8.

14. Wilson JL, Louie BE, Cerfolio RJ, Park BJ, Vallières E, Aye RW, et al. The prevalence of nodal upstaging during robotic lung resection in early stage non-small cell lung cancer. Ann Thorac Surg. 2014;97:1901-6.

15. Society of Thoracic Surgeons. General Thoracic Surgery Database v.2.3. Training manual. Available at: https://www.sts.org/sites/default/files/documents/GTSD trainingmanual_July202018.pdf. Accessed August 15, 2018.

16. Lazar JF, Spier LN, Hartman AR, Lazzaro RS. Standardizing robotic lobectomy: feasibility and safety in 128 consecutive lobectomies within a single healthcare system. Innovations (Phila). 2017;12:77-81

17. Velez-Cubian FO, Rodriguez KL, Thau MR, Moodie CC, Garrett JR, Fontaine JP, et al. Efficacy of lymph node dissection during robotic-assisted lobectomy for non-small cell lung cancer: retrospective review of 159 consecutive cases. $J$ Thorac Dis. 2016;8:2454-63.

18. Veronesi G, Galetta D, Maisonneuve P, Melfi F, Schmid RA, Borri A, et al. Fourarm robotic lobectomy for the treatment of early-stage lung cancer. J Thorac Cardiovasc Surg. 2010;140:19-25.

19. Merritt RE, Hoang CD, Shrager JB. Lymph node evaluation achieved by open lobectomy compared with thoracoscopic lobectomy for N0 lung cancer. Ann Thorac Surg. 2013;96:1171-7.

20. Denlinger CE, Fernandez F, Meyers BF, Pratt W, Zoole JB, Patterson GA, et al Lymph node evaluation in video-assisted thoracoscopic lobectomy versus lobectomy by thoracotomy. Ann Thorac Surg. 2010;89:1730-5.

21. Al-Sarraf N, Aziz R, Doddakula K, Gately K, Wilson L, McGovern E, et al. Fac tors causing inaccurate staging of mediastinal nodal involvement in non-small cell lung cancer patients staged by positron emission tomography. Interact Cardiovasc Thorac Surg. 2007;6:350-3.

22. Decaluwe H, Petersen RH, Brunelli A, Pompili C, Seguin-Givelet A, Gust L, et al. Multicentric evaluation of the impact of central tumour location when comparing rates of N1 upstaging in patients undergoing video-assisted and open surgery for clinical Stage I non-small-cell lung cancer. Eur J Cardiothorac Surg. 2018;53:359-65.

Key Words: robotic, VATS, lobectomy lymph node, upstaging

\section{Discussion}

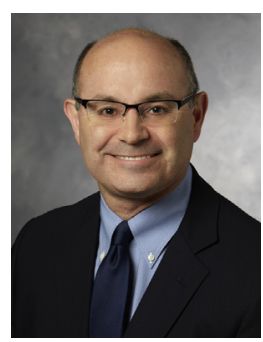

Dr Mark F. Berry (Stanford, Calif). First, I want to congratulate Dr Kneuertz on a great study for a couple of reasons. One is looking at an important issue like lymph node evaluation, which is probably among the most important things that we have in terms of the surgical quality that we provide to our patients beyond just getting a complete resection. I think the more we study this topic the better to make sure that everybody throughout the whole community is doing the best job possible for patients from a cancer standpoint. Two, I know doing a multi-institutional study is a headache in terms of just being able to share data, especially when the data are crossing the ocean. So it's very impressive you were able to do that and increase the size of your cohort. Using pretty sophisticated specific techniques to try to account for the biases that you could see in the study is also very impressive.

My first comment is really based on that potential bias for what you are seeing in the study. Although you controlled for a lot of variables in the analysis, there are a few that you couldn't control for. One that you mentioned was the location, whether it was a central or peripheral location. I think the other thing that you didn't really control for was the specific surgeon who was doing the procedure and whether it was someone who was usually a predominantly open surgeon doing it another way or someone who was usually a robotic surgeon who was doing a video-assisted thoracoscopic surgery (VATS).

So I want to see if you could comment on the selection process for approach in the study. Why were so many patients undergoing open surgery? Why did people choose to do an open approach versus a robotic approach or a VATS approach? For the minimally invasive surgeons, why were they doing VATS over robotic? 


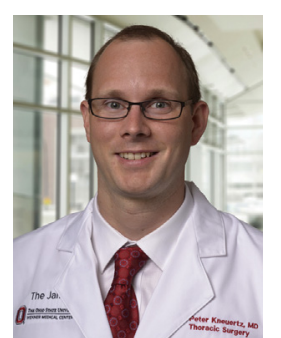

Dr Peter J. Kneuertz (Columbus, Ohio). Thank you, Dr Berry. Those are great comments and they are really important. Certainly this is an effort from 2 institutions, and there are multiple surgeons and each surgeon has his or her own biases. I would say that surgical decision making and the selection of their approach is really intricate and it is hard, as you mentioned, to control for in a retrospective study.

In general, currently we try to apply minimally invasive techniques for all lobectomies for early stage lung cancer. Factors that may lead us to using an open approach would be a very large tumor, chest wall involvement, and I would say bronchoplastic and sleeve resections are still preferably done open, although the robot does help us with that as well.

The study timeline was several years and there was also a dynamic there, so in the early years more open lobectomies were performed more commonly with open thoracotomy.

And then with regard to the selection of 1 minimally invasive approach, VATS versus robotic, I think that has less to do with patients or disease factors and is more a preference. We don't always have the robot available, so that is a factor as well. To accommodate a timely resection, a robotic surgeon may apply a VATS approach for a resection. We had highvolume surgeons at both centers that were early adopters of the robotic technology, and then we had experienced VATS surgeons in both centers as well, but also more junior faculties still in their learning curve with both minimally invasive procedures.

A general trend had been that the robotic approach has been adopted by several VATS surgeons during the study period, and those include more seasoned or senior surgeons.

Dr Berry. That actually leads to my second comment and question, which is basically what is the generalizability of the findings from your study? I think a really good advantage you have in your study is that although you had a lot of patients, you had a limited number of institutions and a limited number of surgeons. That should let you really dig deep, not only through patient characteristics, but also surgeon characteristics in terms of surgeons early in their learning curve or surgeons who are very experienced with 1 approach versus the other. For some of the other studies that you mentioned at the beginning of your talk that looked at registry data or database studies, you don't know anything about those surgeons in terms of what their skill level is or what their experience is.

Although you didn't present data per surgeon, I am sure you have that information. How do you think you can take those data and disseminate them and try to give people advice on what they should do in these situations?

Dr Kneuertz. That is also a great question. Let me start by saying that comparing individual surgeons' data with 2 institutions is a little bit of a political problem, so we didn't dig too deep into that. I think a very interesting analysis that we did is for those surgeons who were VATS surgeons who converted to robotics, which includes the senior author Dr Merritt, who was an experienced VATS surgeon before he switched to the robot. We analyzed their data. and found that for him at least the number of lymph nodes removed increased with the robot, but there was no difference in the lymph node upstaging, and that may be because of the same approach that he takes to the lymph nodes. But I think a general observation is that the robot reduces the effort to do a very good lymph node dissection.

Our data cannot be generalized, and I think we have to be cautious with retrospective data. The results reflect our practices and our patients, which may not be transferrable to other centers.

For example, granulomatous lymph node disease was high in our patient population and that affects the lymph node dissection. However, if we performed this study in other centers, we would likely make similar observations, because the results reflect the surgeons' experience with the approaches. 


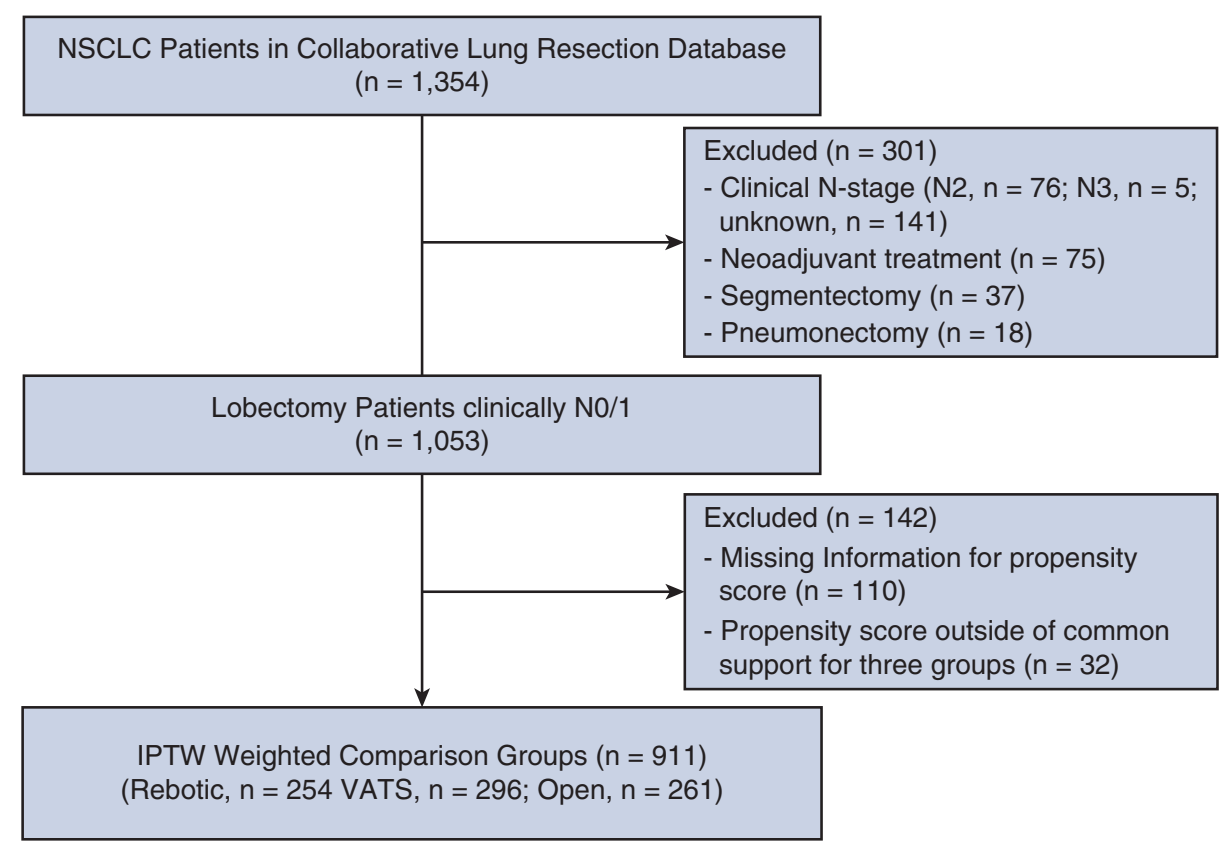

FIGURE E1. Consolidated Standards of Reporting Trials flowsheet outlining the selection of the study cohort. NSCLC, non-small cell lung cancer; IPTW, inverse probability of treatment weight; VATS, video-assisted thoracoscopic surgery.

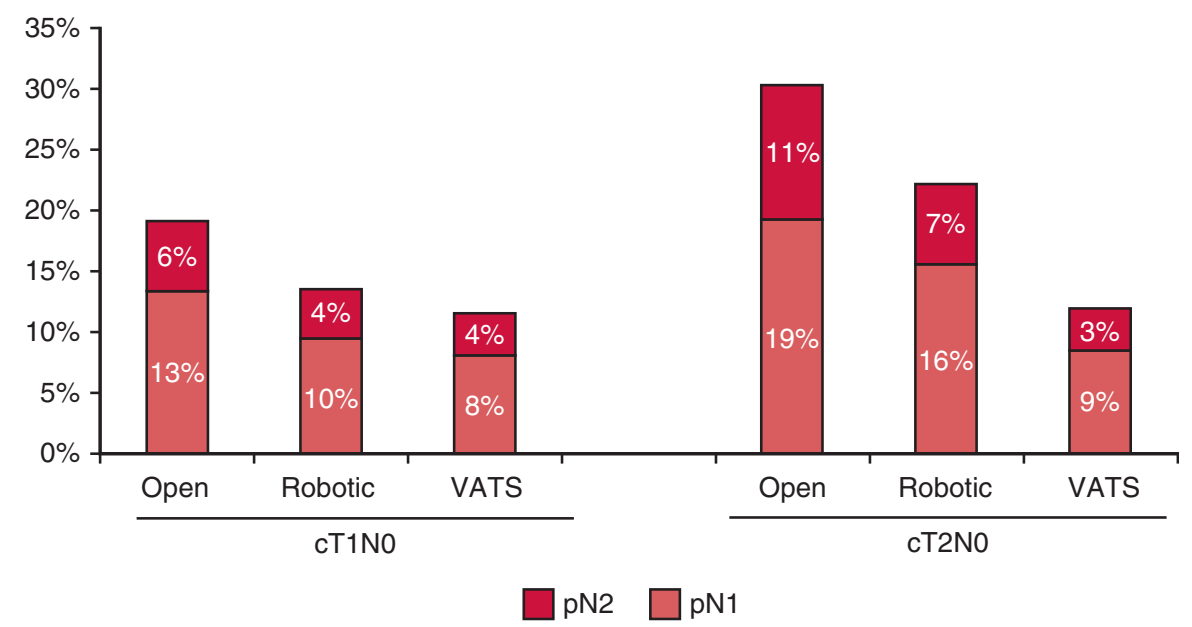

FIGURE E2. Inverse probability of treatment weight (IPTW) adjusted rates of N1 and N2 lymph node upstaging of clinically node negative non-small cell lung cancer following open, robotic, and video-assisted thoracoscopic surgery (VATS) lobectomy increase proportionally between clinical stage T1 and T2 tumors. 
TABLE E1. Comparison of preoperative staging modalities and operative approach by institution

\begin{tabular}{lrrr}
\hline \multicolumn{1}{c}{ Variable } & $\begin{array}{c}\text { Center 1 } \\
(\mathbf{n = 3 4 4 )}\end{array}$ & $\begin{array}{c}\text { Center 2 } \\
(\mathbf{n = 7 0 9 )}\end{array}$ & $\boldsymbol{P}$ value \\
\hline $\begin{array}{l}\text { Staging modality } \\
\quad \text { PET scan }\end{array}$ & $208(61.0)$ & $668(94.2)$ & $<.001$ \\
$\quad$ EBUS & $204(59.3)$ & $121(17.1)$ & $<.001$ \\
$\quad$ EBUS or mediastinoscopy & $215(62.5)$ & $153(21.7)$ & $<.001$ \\
Operative approach & & & \\
$\quad$ Robotic & $37(10.8)$ & $354(49.9)$ & $<.001$ \\
$\quad$ VATS & $162(47.1)$ & $176(24.8)$ & \\
$\quad$ Open & $145(42.2)$ & $179(25.3)$ & \\
\hline
\end{tabular}

Values are presented as $\mathrm{n}(\%) . P E T$, Positron emission tomography; EBUS, endobronchial ultrasound; VATS, video-assisted thoracoscopic surgery.

TABLE E2. Inverse probability of treatment weight-adjusted comparison of total number of lymph node stations $(\mathrm{N} 1+\mathrm{N} 2)$ assessed by approach

\begin{tabular}{|c|c|c|c|c|}
\hline No. & $\begin{array}{c}\text { Robotic } \\
(n=254)\end{array}$ & $\begin{array}{c}\text { VATS } \\
(\mathrm{n}=\mathbf{2 9 6})\end{array}$ & $\begin{array}{c}\text { Open } \\
(\mathrm{n}=\mathbf{2 6 1})\end{array}$ & $P$ value \\
\hline 0 & 1.1 & 0 & 0.5 & \\
\hline 1 & 0.7 & 1.2 & 2.6 & \\
\hline 2 & 7.7 & 13.3 & 13.3 & \\
\hline 3 & 18.3 & 19.6 & 31.6 & \\
\hline 4 & 34.7 & 34 & 29.7 & \\
\hline 5 & 30.9 & 27.4 & 17.9 & \\
\hline 6 & 6.6 & 4.1 & 3.2 & \\
\hline 7 & 0.2 & 0.5 & 1.2 & \\
\hline Total & 100 & 100 & 100 & \\
\hline Mean & 3.8 & 3.6 & 4.0 & .001 \\
\hline $\begin{array}{l}95 \% \text { Confidence } \\
\text { interval }\end{array}$ & $3.7-4.0$ & $3.4-3.7$ & $3.8-4.0$ & \\
\hline
\end{tabular}

1466.e2 The Journal of Thoracic and Cardiovascular Surgery • November 2019 